\title{
HALLERVORDEN-SPATZ DISEASE: CLINICOPATHOLOGY
}

Clinical and pathological features of familial late infantile Hallervorden-Spatz disease (HSD) are reported in two sisters, one of whom died at 11 years, from the Institute for Neurological Sciences, University of Siena, Italy. Clinical diagnosis was confirmed by the classical "eye of the tiger" sign in the MRI. The appearance of the globus pallidus on MRI correlated with the pathological findings, showing pallidal axonal spheroids and iron deposits without involvement of the substantia nigra. Clinically, retinitis pigmentosa, acanthocytosis, and neuromuscular involvement with increased serum creatine kinase were observed in both patients. HSD is classified as a form of neuroacanthocytosis, along with choreo-acanthocytosis, McLeod syndrome, and HARP syndrome. These diseases have the following clinical features in common but variable in frequency: 1) acanthocytosis, 2) extrapyramidal movements, 3) neuromuscular involvement, and 4) retinitis pigmentosa. (Malandrini A et al. Clinicopathological study of familial late infantile Hallervorden-Spatz disease: a particular form of neuroacanthocytosis. Child's Nerv Syst March 1996;12:155-160). (Respond: Dr A Malandrini, Institute for Neurological Sciences, University of Siena, Viale Bracci, 2, I-53100 Siena, Italy).

COMMENT. Hallervorden-Spatz disease is a rare, progressive, and fatal degenerative disorder, with onset in late infancy, childhood or adulthood, characterized by a bizarre gait and speech disturbance, dystonic postures and choreo-athetotic movements, mental deterioration, retinitis pigmentosa, and occasionally, acanthocytosis. The coexistence of HSD and acanthocytosis in 3 sisters was reported by Swisher CN, Menkes JH, Cancilla PA and Dodge PR. Trans Am Neurol. Assoc 1972;97:212. An autosomal recessive inheritance is suggested by familial cases. Diagnosis may be confirmed by the MRI and the "eye of the tiger sign" affecting the globus pallidus. For further reviews of HSD and related disorders, see Ped Neur Briefs Nov 1995;9:85, and Progress in Pediatric Neurology II, PNB Publ, 1994, p 477.

\section{MUSCLE DISORDERS}

\section{DIAGNOSIS OF CONGENITAL MUSCULAR DYSTROPHY}

Patterns of alkaline and acid phosphatases were compared with the distribution of merosin and dystrophin staining in muscle biopsies from 20 children with congenital muscular dystrophy (CMD) examined at the Department of Neurology, Washington University School of Medicine, St Louis, MO. A ratio of AcP:AlkP staining was calculated for each biopsy. In 9 patients with CMD with normal dystrophin, the AcP:AlkP ratio was low, whereas in 3 patients with CMD and reduced dystrophin and in 7 with Duchenne muscular dystrophy, the ratio was up to 15 times higher. Low AcP:AlkP ratios were correlated with absence of AcP-positive cells. Merosin staining was absent in 5 of 17 CMD patients, none of whom could walk, whereas all 12 with merosinpositive stains walked. (Connolly AM, Pestronk A, et al. Congenital muscular dystrophy syndromes distinguished by alkaline and acid phosphatase, merosin, and dystrophin staining. Neurology March 1996;46:810-814). (Respond: Dr Alan Pestronk, Department of Neurology, Box 8111, Washington University School of Medicine, 660 S Euclid Ave, St Louis, MO 63110).

COMMENT. Biopsies showing few acid phosphatase-positive cells in association with numerous alkaline phosphatase staining muscle fibers are 
specific for congenital muscular dystrophy syndromes and histopathological support for the diagnosis. A finding of reduced merosin in muscle is predictive of severe weakness and disability.

Classical (Occidental) Merosin-positive form of CMD was milder and more slowly progressive than the merosin-negative form and Fukuyama type in a clinical and pathological study of 50 patients examined at the National Institute of Neuroscience, National Center of Neurology and Psychiatry, Kodaira, Tokyo, Japan. (Kobayashi O et al. Congenital muscular dystrophy: Clinical and pathological study of 50 patients with the classical (Occidental) merosin-positive form. Neurology March 1996;46:815-818).

Cognitive dysfunction in Becker's muscular dystrophy was the major presenting feature in 4 patients reported from the Children's Hospital, Boston, the Texas Children's Hospital, Houston, and other centers. (North KN, Miller G et al. Neurology March 1996;46:461-465). Psychiatric disturbance was also a feature in the absence of muscle weakness. An elevated serum creatine kinase may provide a valuable screening test in boys with unexplained cognitive or psychiatric disturbance. One patient had received various drugs used for ADHD before diagnosis was determined.

\section{LEARNING DISABILITIES}

\section{ARTICULATORY FEEDBACK DEFICIT IN DYSLEXIA}

An efferent or "motor-articulatory feedback" hypothesis for developmental dyslexia-phonological type is proposed from the Departments of Neurology and Psychiatry, University of Florida College of Medicine, Gainesville, FL. Most children learn to read by the alphabetic system, requiring phonological awareness and conversion of letters (graphemes) into speech sounds (phonemes). Most dyslexics have deficient phonological awareness and difficulty converting graphemes into phonemes. The left inferior frontal lobe is important in phonological reading, as suggested by patients with acquired lesions and PET studies of normal subjects. Dyslexic children are unable to perceive the position and movement of the articulatory apparatus (mouth, lips, tongue) during speech, impairing phonological awareness and conversion of graphemes to phonemes. Deficits in motorarticulatory programming or feedback may be related to this lack of awareness of articulators. (Heilman KM et al. Developmental dyslexia: a motorarticulatory feedback hypothesis. Ann Neurol March 1996:39:407-412). (Respond: Dr Kenneth M Heilman, Box 100236, University of Florida, Gainesville, FL 32610).

COMMENT. Other current hypotheses for developmental dyslexia are 1) visual hypothesis, with dysfunction in the visual perception system, and 2) auditory hypothesis, with abnormalities in the rapid discrimination of lowcontrast, complex sounds and associated speech and language disturbances.

A disconnection syndrome hypothesis for developmental dyslexia is proposed based on evidence from PET scanning studies conducted at the MRC Cognitive Development Unit, London, UK. (Paulesu E, Frith U et al. Brain Feb 1996;119:143-157). A rhyming and a short-term memory task with visually presented letters was used to study brain activity in 5 compensated adult developmental dyslexics. Brain regions normally activated in phonological processing were defective, and weak connections between anterior and posterior language areas are proposed. 